\title{
FOLIA 233
}

\section{Annales Universitatis Paedagogicae Cracoviensis Studia Mathematica XVII (2018)}

\section{Karol Gryszka On asymptotically periodic-like motions in flows}

Communicated by Justyna Szpond

\begin{abstract}
We study three properties associated to the recurrence of orbits in flows: asymptotic periodicity, positive asymptotic periodicity and G-asymptotic periodicity. We determine which implications between these notions hold and which do not. We also show how these notions are related to Lyapunov stability.
\end{abstract}

\section{Introduction}

Non-periodic orbits in flows (dynamical systems with continuous time) may still reveal some kind of periodic-like recurrence (see [2, 3, 4, 5, 10, 11]; also [1] for an overview). This kind of behaviour may occur "asymptotically", i.e. when the time approaches infinity. It can also occur in a way that can be detected already in finite time. Interestingly, although similar in spirit, the relevant notions may have quite different consequences, for example some of them lead to bounded orbits, while some other do not. The impact of asymptotic periodic-like recurrence on the form of omega-limit sets has been studied in [8, 9] (see also [6] and the references therein).

In this paper we investigate and compare three particular notions that capture periodic-like recurrence: asymptotic periodicity, positive asymptotic periodicity and G-asymptotic periodicity, as introduced in [6], [9] and [7, respectively. We will show which implications between these notions hold, and we will give counterexamples for implications that fail. We are also interested in relations between the above three notions and Lyapunov stability, as defined and studied in [1, 6].

AMS (2010) Subject Classification: Primary: 37C25, 34D05; Secondary: 34C99, 37C75.

Keywords and phrases: periodicity, asymptotic periodicity, stability. 


\section{Preliminaries}

We introduce basic definitions and notations. Throughout the paper $(X, d)$ is a metric space with a metric $d$.

A dynamical system (a flow) $\phi$ is a continuous mapping $\phi: \mathbb{R} \times X \rightarrow X$ such that $\phi(0, x)=x$ and $\phi(t, \phi(s, x))=\phi(t+s, x)$ for any $x, s$ and $t$ belonging to the respective sets. We call $X$ the phase space of $\phi$ and interpret $t$ as the time. The motion through $x$ is the mapping $t \mapsto \phi(t, x)$. We will often attribute the properties of the motion through $x$ with the point $x$ itself. Given a dynamical system $\phi$ and $x \in X$, the set $o(x)=\phi(\mathbb{R}, x)$ is the orbit of $x$ and $o^{+}(x)=\phi([0,+\infty), x)$ is the positive orbit of $x$. A point $x$ is stationary if $x=\phi(t, x)$ for any $t \in \mathbb{R}$. If for some $T>0$ we have $x=\phi(T, x)$ and $x$ is not stationary, then $x$ is periodic. If $T>0$ is the smallest such that $x=\phi(T, x)$, then we say that $x$ is T-periodic and call $T$ the period of $x$. The $\omega$-limit set $\omega(x)$ consists of all points $y \in X$ such that there exists a strictly increasing and diverging to $+\infty$ sequence $\left(t_{n}\right)_{n \in \mathbb{N}}$ of times with the property that $\lim _{n \rightarrow+\infty} \phi\left(t_{n}, x\right)=y$. By changing the direction of time in the latter definition we define the $\alpha$-limit set $\alpha(x)$.

We now introduce the most important definitions for the paper. For this purpose we fix a dynamical system $\phi$ on a metric space $(X, d)$.

\subsection{Asymptotically periodic motions}

The following definition comes from [6].

DEFINITION 2.1

The motion through $x$ is called asymptotically T-periodic (resp. asymptotically stationary) if there exists a $T$-periodic (resp. stationary) point $p$ such that

$$
\lim _{t \rightarrow+\infty} d(\phi(t, x), \phi(t, p))=0
$$

\subsection{Positively asymptotically periodic motions}

The following definition is due to Pelczar $([9])$. An important feature of the notion is that it generalizes the previous definition (see Proposition 3.1) without requiring the presence of any limit set.

DEFINITION 2.2

Assume that $x \in X$ is not a stationary point. We say that $x$ is positively asymptotically periodic if there exists a $T>0$ such that for any $\varepsilon>0$ there is an $s \geq 0$ such that if $t \geq s$, then $d(\phi(t+T, x), \phi(t, x))<\varepsilon$.

\subsection{G-asymptotically periodic motions}

The following definition is based on the asymptotic behaviour of the orbit outside of a small neighbourhood of a point belonging to the positive orbit of $x$. This idea was introduced in [7]. We precede this definition by introducing some necessary notation. 
Fix $x \in X$ and $\varepsilon>0$, and define the set

$$
A(x, \varepsilon):=\{t \geq 0: d(\phi(t, x), x)>\varepsilon\} .
$$

This set is the union of at most countably many pairwise disjoint and open intervals denoted by $\left(q_{i}, r_{i}\right)$, where the set of indices is either finite (the case where $r_{i}=+\infty$ for some $i$ ) or countable. Define

$$
w_{x, \varepsilon}(t):= \begin{cases}0, & t \notin A(x, \varepsilon), \\ \left|r_{i}-q_{i}\right|, & t \in\left(q_{i}, r_{i}\right) .\end{cases}
$$

The set $W_{x, \varepsilon}:=\left\{w_{x, \varepsilon}(t): t \geq 0\right\}$ contains non-negative real numbers, including $+\infty$ if necessary. Set

$$
W(x, \varepsilon):=\limsup _{t \rightarrow+\infty} w_{x, \varepsilon}(t) .
$$

DEFINITION 2.3

The $G$-asymptotic period of $x$ (of the orbit of $x$ ) is defined as

$$
\operatorname{GAP}(x):=\lim _{\varepsilon \rightarrow 0} \limsup _{t \rightarrow+\infty} W(\phi(t, x), \varepsilon) .
$$

If $\operatorname{GAP}(x)=0$, then $x$ is called $G$-asymptotically fixed. If $x$ has a finite asymptotic period, then it is called G-asymptotically periodic. If $\operatorname{GAP}(x)=+\infty$, then a point $x$ is called G-asymptotically non-periodic.

Originally, the author of [7] named G-asymptotically periodic points asymptotically periodic. However, to avoid confusion with other notions we add the prefix "G-" to highlight that the notion generalizes asymptotically periodic motions.

Let us note that $\mathrm{G}$-asymptotic periodicity is the orbit property, that is if $z \in o(x)$, then $\operatorname{GAP}(z)=\operatorname{GAP}(x)$.

Note that all the definitions can be reformulated by changing the time direction towards $-\infty$. This procedure allows to define the negative counterpart. We do not

investigate that case as it is similar to the one with positive time and all results carry over with obvious modifications.

\subsection{Stability}

We recall the definition of stability. The motion through $x$ is positively Lyapunov stable in a subset $N$ of $X$ if for any $\varepsilon>0$ there is a $\delta>0$ such that $y \in N \cap B(x, \delta)$ implies $d(\phi(t, x), \phi(t, y))<\varepsilon$ for $t \geq 0$. Similarly, it is negatively Lyapunov stable (resp. Lyapunov stable in both directions) if the above condition is satisfied with $t \leq 0$ (resp. $t \in \mathbb{R}$ ). If in the definition we take $N$ as a neighborhood of $x$, then we can delete "in a subset $N$ of $X$ ". In such a case the motion through $x$ is positively Lyapunov stable (resp. negatively Lyapunov stable, Lyapunov stable in both directions) if for any $\varepsilon>0$ there is a $\delta>0$ such that if $d(x, y)<\delta$, then $d(\phi(t, x), \phi(t, y))<\varepsilon$ for $t \geq 0$ (resp. $t \leq 0, t \in \mathbb{R})$. 


\section{Investigating mutual relations}

We begin with a straightforward relation between asymptotic $T$-periodicity and positive asymptotic periodicity.

Proposition 3.1

Let $\phi$ be a dynamical system on a metric space $(X, d)$. If the motion through $x$ is asymptotically T-periodic, then it is positively asymptotically periodic.

Proof. This follows immediately from the definition. Let $p \in X$ be a $T$-periodic point taken from the definition of asymptotic $T$-periodicity and let $\varepsilon>0$. There exists an $s>0$ such that $d(\phi(t, x), \phi(t, p))<\frac{\varepsilon}{2}$ for all $t>s$. We have $d(\phi(T+$ $t, p), \phi(t, p))=0$, thus for $t>s$,

$$
\begin{aligned}
d(\phi(T+t, x), \phi(t, x)) \leq & d(\phi(T+t, x), \phi(T+t, p))+d(\phi(T+t, p), \phi(t, p)) \\
& +d(\phi(t, p), \phi(t, x)) \\
& <\frac{\varepsilon}{2}+\frac{\varepsilon}{2}=\varepsilon .
\end{aligned}
$$

The converse to the above theorem does not hold. This is because positively asymptotically periodic orbits need not be bounded - see Example 3.4 and Proposition 3.5

Before we prove that G-asymptotic periodicity is weaker than asymptotic periodicity, we need to establish the following supplementary lemma.

LEMMA 3.2

Let $\phi$ be a dynamical system on a metric space $(X, d)$ and let $x \in X$. If $\operatorname{GAP}(x)=$ 0 , then the set $\omega(x)$ consists of at most one point.

Proof. The assertion follows from Theorem 2.2 in [7] (the implication $(\Rightarrow)$ in that theorem holds true for general metric spaces).

THEOREM 3.3

Let $\phi$ be a dynamical system on a metric space $(X, d)$. If $x \in X$ is asymptotically $T$-periodic, then it is $G$-asymptotically periodic.

Proof. Let $p$ be taken from the definition of asymptotic $T$-periodicity. Then

$$
d(\phi(k T, x), p)=d(\phi(k T, x), \phi(k T, p)) \rightarrow 0 \quad(k \rightarrow+\infty)
$$

and the sequence $(\phi(k T, x))_{k \in \mathbb{N}}$ converges to $p$. By the convergence, there is a $K>0$ such that if $k \geq K$, then $d(\phi(k T, x), p)<\frac{\varepsilon}{3}$. On the other hand, there is an $A>0$ such that if $t \geq A$, then $d(\phi(t, x), \phi(t, p))<\frac{\varepsilon}{3}$. Since $p$ is $T$-periodic, taking $t \geq A$ and $k \geq K$ we conclude the following

$$
d(\phi(k T+t, x), \phi(t, p))=d(\phi(k T+t, x), \phi(k T+t, p))<\frac{\varepsilon}{3} .
$$


Consider any $u_{k, t}:=k T+t$, where $k \geq K$ and $t \geq A$. Note that $u_{k, t}$ defined in that way can describe any sufficiently large value of time. We claim that for such $u_{k, t}$ we have $r_{i}-q_{i} \leq T$. Indeed, if we consider the ball $B\left(\phi\left(u_{k, t}, x\right), \varepsilon\right)$, then $\phi\left(l T, \phi\left(u_{k, t}, x\right)\right) \in B\left(\phi\left(u_{k, t}, x\right), \varepsilon\right)$ for any $l \in \mathbb{N}$. In other words, if the orbit of $\phi\left(u_{k, t}, x\right)$ leaves the ball, it must return for some time $t \leq T$. We have just proven that for any $\varepsilon>0$ there is some $s>0$ such that for any $t>s$ we have $W(\phi(t, x), \varepsilon) \leq T$ and therefore $\lim \sup _{t \rightarrow+\infty} W(\phi(t, x), \varepsilon) \leq T$. As a consequence, $\operatorname{GAP}(x)$ is finite.

By Lemma 3.2 , if $\operatorname{GAP}(x)=0$, then the set $\omega(x)$ is either empty or consists of stationary point. In either case it contradicts the periodicity of $p$.

The key factor in Proposition 3.1 and Theorem 3.3 is the presence of a periodic orbit. However, G-asymptotically periodic orbits can also have empty limit sets see Example 3.6 and Proposition 3.7 .

We now present two examples that were announced earlier.

EXAMPLE 3.4

Consider a dynamical system $\phi$ on the plane. It is defined to have its trajectories coinciding with the curves in polar coordinates

$$
\begin{aligned}
& r(t)= \begin{cases}\ln t & \text { for } t \geq e, \\
e^{t-e} & \text { for } t<e,\end{cases} \\
& \theta(t)=t+c,
\end{aligned}
$$

where $c \in \mathbb{R}$. Note that the $\alpha$-limit set of any orbit is the set $\{(0,0)\}$ consisting of a stationary point. In this setting any orbit (of a point that is not stationary) intersects a given half-line $A x+B y=0$ ( $A$ and $B$ are fixed) originating at $(0,0)$ at equal time gaps $2 \pi$.

\section{Proposition 3.5}

The dynamical system $\phi$ constructed in Example 3.4 contains a positively asymptotically periodic point whose orbit is unbounded. In particular, there is a motion that is positively asymptotically periodic but neither is asymptotically T-periodic for any value of $T$ nor $G$-asymptotically periodic.

Proof. We limit the reasoning to sufficiently large times $t$ and exclude the stationary point, so that only logarithmic parts of the curves are considered.

Without loss of generality we pick the curve with $c=0$. In Cartesian coordinates we have

$$
\left\{\begin{array}{l}
x(t)=\cos t \ln t, \\
y(t)=\sin t \ln t .
\end{array}\right.
$$

Let us compare the distance between two points in this trajectory at the time distance $2 \pi$,

$$
\left(d((x(t+2 \pi), y(t+2 \pi)),(x(t), y(t)))^{2}=\ln ^{2}(2 \pi+t)-\ln ^{2}(t) \rightarrow 0 \quad(t \rightarrow+\infty) .\right.
$$


It follows that the definition of positively asymptotically periodic motion is satisfied for $T=2 \pi$. It is clear that the same property holds for any not stationary point on the plane.

Clearly, the orbit described above is not bounded hence no other property described in the Proposition holds.

EXAMPLE 3.6

Consider the following sequences of numbers:

$$
\begin{aligned}
& a_{n}:=2 \pi n-\frac{1}{n}, \quad b_{n}:=2 \pi n-\frac{1}{n+1}, \quad c_{n}:=2 \pi n+\frac{1}{n+1}, \\
& d_{n}:=2 \pi n+\frac{1}{n}, \quad e_{n}:=\pi(2 n+1)
\end{aligned}
$$

and the sequence of intervals

$$
A_{n}:=\left[a_{n}, d_{n}\right] .
$$

Define a function $f: \mathbb{R} \rightarrow \mathbb{R}$ by the following rule. If $t$ is not in any $A_{n}$, set $f(t)=1$. For any $n$, define $f$ on $A_{n}$ to be a piecewise linear function joining the points $\left(a_{n}, 1\right),\left(b_{n}, 2\right),\left(c_{n}, 2\right),\left(d_{n}, 1\right)$. These linear parts will be called "hills" (see Figure 11.

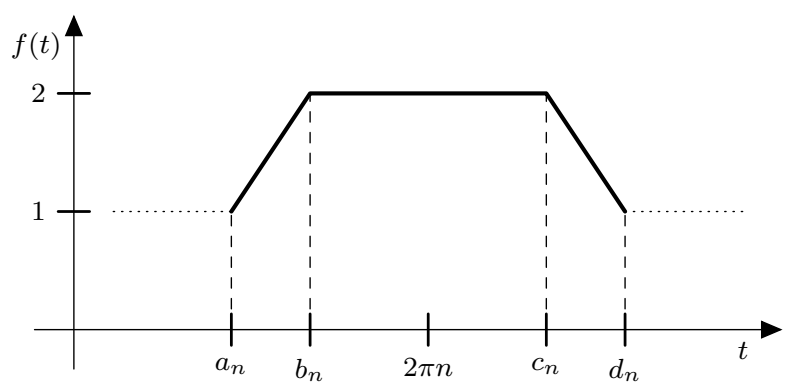

Figure 1: The hill of $f$ on the interval $A_{n}$.

The function $f$ we have just defined has the following key property:

for any $n$ we have $f\left(b_{n}\right)=2$ and $f\left(b_{n}+2 \pi\right)=f\left(a_{n+1}\right)=1$.

Define the curve in $\mathbb{R}^{3}$ using the following description

$$
\Xi: \mathbb{R} \ni t \mapsto\left(f(t) \cos t, f(t) \sin t, e^{-t}\right) \in \mathbb{R}^{3} .
$$

This parametric representation gives the following intuition. The graph of $f$ is wrapped around the upper half of the cylinder $x^{2}+y^{2}=1$ with the coils converging decreasingly to the plane $z=0$. The hills stick away from the cylinder with their middle points projecting to the point $(2,0,0)$ (see Figure 2). 
The dynamical system $\phi$ is defined only in the curve $\Xi$. The motion occurs towards the plane $z=0$ with a constant angular velocity equal to 1 . In particular, the following are true:

- if $(t, x)$ is such that $\phi(t, x)=\Xi\left(e_{n}\right)$ for some $n$, then $\phi(t+2 \pi, x)=\Xi\left(e_{n+1}\right)$,

- if $(t, x)$ is such that $\phi(t, x)=\Xi\left(b_{n}\right)$ for some $n$, then $\phi(t+2 \pi, x)=\Xi\left(a_{n+1}\right)$.

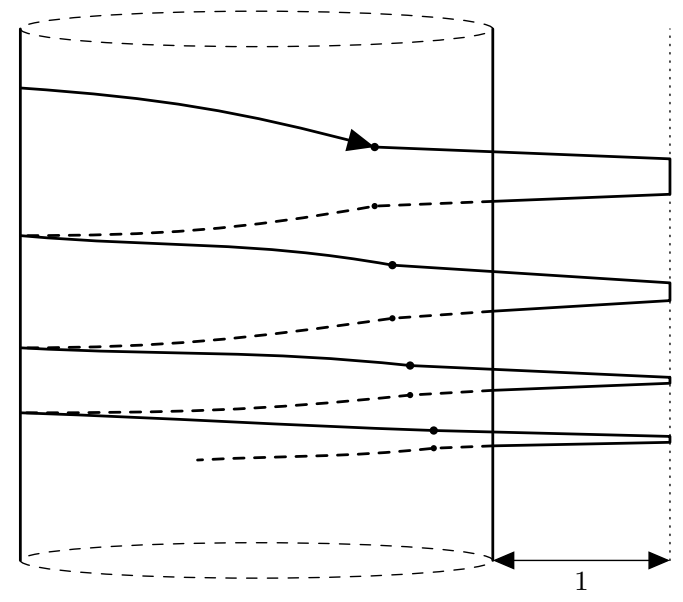

Figure 2: The sketch of the orbit.

\section{Proposition 3.7}

The dynamical system $\phi$ constructed in Example 3.6 contains a point that is $G$ asymptotically periodic but neither is positively asymptotically periodic nor asymptotically $T$-periodic for any value of $T$.

Proof. To show that the only orbit of $\phi$ is G-asymptotically periodic we take any $\varepsilon>0$ and consider a point $y$ in the trajectory such that the intersection of $B(y, \varepsilon)$ with the plane $z=0$ is not empty.

Note that from the definition of the function $f$ and the system $\phi$ it follows that the return time in the above situation is always bounded by $2 \pi$. Hence corresponding limits are bounded by $2 \pi$. Since the same argument follows for any sufficiently small $\varepsilon$ and sufficiently large $t$ such that $y=\phi(t, x)$ satisfies the above description, we conclude that $\operatorname{GAP}(x) \leq 2 \pi$.

We can also prove that the above inequality is in fact an equality. The idea is that as we go with $\varepsilon$ to 0 , it follows from the construction of $\Xi$ that the difference $r_{i}-q_{i}$, by the constant angular velocity, converges to $2 \pi$. We omit the rather technical proof.

Let us now prove that the motion through $x$ is not positively asymptotically periodic. We divide the argument into three cases. 
Case 1. Assume that $T=2 \pi \cdot n+a$ for some $n \geq 0$ and $a \in(0,2 \pi)$ is such that the definition of positively asymptotically periodic motion holds for $T$. Denote

$$
b:=\inf \left\{d\left((-1,0,0), \Xi\left(e_{k}+a\right)\right): k \in \mathbb{N}\right\} .
$$

Then $b>0$. Take any $\varepsilon<\frac{b}{3}$ and let $s$ be such that for $t>s$ we have $d(\phi(t+$ $T, x), \phi(t, x))<\varepsilon$. Furthermore, we can find a sufficiently large $t^{\prime}>s$ and a $k>0$ such that $\phi\left(t^{\prime}, x\right)=\Xi\left(e_{k}\right)$ and $d\left(\Xi\left(e_{k}\right),(-1,0,0)\right)<\frac{b}{3}$. Then $\phi\left(t^{\prime}+T, x\right)=$ $\Xi\left(e_{n+k}+a\right)$ and

$$
d\left(\phi\left(t^{\prime}, x\right), \phi\left(t^{\prime}+T, x\right)\right)=d\left(\Xi\left(e_{k}\right), \Xi\left(e_{n+k}+a\right)\right) \geq \frac{b}{3},
$$

a contradiction.

Case 2. Assume that $T=2 \pi$ is such that the definition of positively asymptotically periodic motion holds for $T$. Denote

$$
b:=\inf \left\{d\left(\Xi\left(b_{k}\right), \Xi\left(a_{k+1}\right)\right): k \in \mathbb{N}\right\} .
$$

Then $b>0$. Take any $\varepsilon<b$ and let $s$ be taken from the definition of positively asymptotically periodic motion. Take any large $t^{\prime}>s$ such that $\phi\left(t^{\prime}, x\right)=\Xi\left(b_{k}\right)$ for some $k \in \mathbb{N}$. Then $\phi\left(t^{\prime}+2 \pi, x\right)=\Xi\left(a_{k+1}\right)$ and

$$
d\left(\phi\left(t^{\prime}, x\right), \phi\left(t^{\prime}+T, x\right)\right)=d\left(\Xi\left(b_{k}\right), \Xi\left(a_{k+1}\right)\right) \geq b>\varepsilon,
$$

a contradiction.

Case 3. Assume that $T=2 \pi \cdot n$ for $n>0$. The reasoning is analogous as in the Case 2.

In conclusion, we have proven that no value of $T$ can be used in the definition, therefore the motion cannot be positively asymptotically periodic. Clearly, the asymptotic T-periodicity property cannot hold due to the absence of the limit set.

We are ready to summarize the relations obtained in this section.

\section{THEOREM 3.8}

The implications not following from the diagram do not hold.

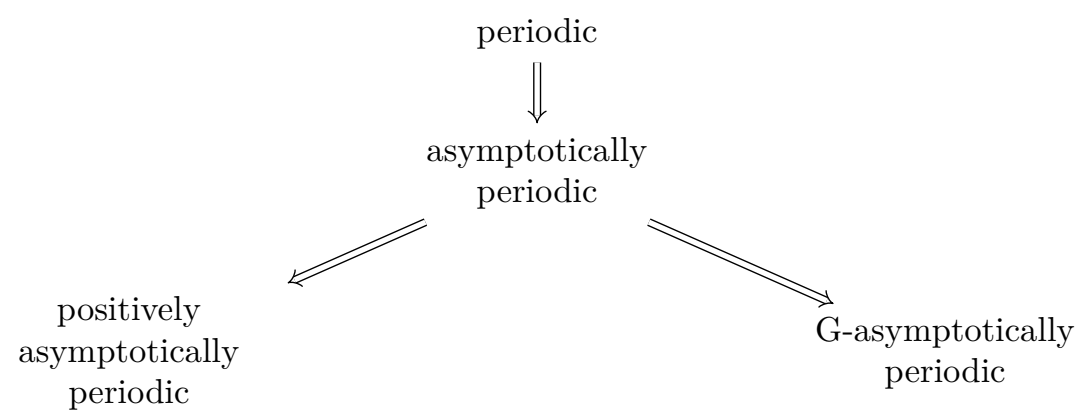




\section{The role of stability}

The notion of Lyapunov stability plays a significant role in determining whether a motion satisfies asymptotically periodic-like properties. The following classic results express a sufficient condition for such a behaviour.

THEOREM 4.1 ( $[9])$ Let $\phi$ be a dynamical system on a metric space $(X, d)$ and $y \in \omega(x)$. If $y$ is stationary or periodic and positively Lyapunov stable, then $x$ is positively asymptotically periodic. 4.5 .

We will consider an improved version of the latter theorem later (see Theorem

THEOREM $4.2([6])$

Let $\phi$ be a dynamical system on a metric space $(X, d)$. The motion through $x$ is asymptotically stationary (resp. asymptotically T-periodic) if and only if the following conditions hold:

1. $x$ is $L^{+}$-stable, that is the set $\overline{o^{+}(x)}$ is compact,

2. $o^{+}(x)$ is uniformly Lyapunov stable in the positive direction with respect to the set $o^{+}(x)$, that is for any $\varepsilon>0$ there is a $\delta>0$ such that if $u, v \in o^{+}(x)$ and $d(u, v)<\delta$, then $d(\phi(t, u), \phi(t, v))<\varepsilon$ for any $t \geq 0$,

3. the set $\omega(x)$ is a stationary point (resp. T-periodic orbit).

We now prove a result regarding G-asymptotically periodic motions. Surprisingly, the Lyapunov stability is so strong that it implies that the motion is both positively asymptotically periodic and G-asymptotically periodic, even though these two notions are, according to Theorem 3.8, not related to each other.

\section{THEOREM 4.3}

Let $\phi$ be a dynamical system on a metric space $(X, d)$ and let $y \in \omega(x)$. If $y$ is stationary or periodic and positively Lyapunov stable, then $x$ is G-asymptotically stationary or G-asymptotically periodic.

Proof. To prove the assertion we first assume that $y$ is stationary. Fix $\varepsilon>0$ and take $0<\delta<\frac{\varepsilon}{4}$, so that the following condition is satisfied (compare with Lyapunov stability)

$$
d(y, v)<\delta \text { implies } d(\phi(t, y), \phi(t, v))<\frac{\varepsilon}{2} \quad \text { for each } t>0 .
$$

Since $y \in \omega(x)$ we find a $t_{0}$ such that $d\left(\phi\left(t_{0}, x\right), y\right)<\delta$. Denote $z=\phi\left(t_{0}, x\right)$. Then, since $d(z, y)<\delta$, the stability condition ensures that $d(y, \phi(t, z))<\frac{\varepsilon}{2}$. In particular, $o^{+}(z) \subset B(z, \varepsilon)$. The described inclusion holds for any $\varepsilon$ and sufficiently large $t_{0}$. We conclude that $\operatorname{GAP}(x)=0$.

We now consider the case of $T$-periodic point. It is sufficient to show that $\operatorname{GAP}(x)$ is finite and positive. Take a sequence $\left(t_{n}\right)_{n \in \mathbb{N}}$ according to the relation $y \in \omega(x)$ and without loss of generality assume that $z=\phi\left(t_{0}, x\right)$ is such that 
$\phi\left(t_{n}, z\right) \rightarrow y$ and $d(z, y)<\delta$. The number $\delta$ is chosen in such a way that for a given $\varepsilon$ the following stability condition holds

$$
d(y, v)<\delta \text { implies } d(\phi(t, y), \phi(t, v))<\frac{\varepsilon}{2} \quad \text { for each } t>0 .
$$

Consider any natural number $k$. Then

$$
\phi\left(t_{n}+k T, z\right)=\phi\left(k T, \phi\left(t_{n}, z\right)\right) \rightarrow \phi(k T, y)=y \quad(n \rightarrow+\infty) .
$$

In particular, there is an $N \geq 0$ such that for each $n \geq N, d\left(\phi\left(t_{n}+k T, z\right), y\right)<\delta$. Note that the above property need not be true for every $n \in \mathbb{N}$. Using stability again we conclude that for any $s>0$,

$$
d\left(\phi\left(t_{n}+k T+s, z\right), \phi(s, y)\right)<\frac{\varepsilon}{2} .
$$

Note that $t_{n}+k T+s$ can describe any sufficiently large real number. By the above we have

$$
\begin{aligned}
d\left(\phi(s, z), \phi\left(t_{n}+k T+s, z\right)\right) & \leq d(\phi(s, z), \phi(s, y))+d\left(\phi(s, y), \phi\left(t_{n}+k T+s, z\right)\right) \\
& <\frac{\varepsilon}{2}+\frac{\varepsilon}{2}=\varepsilon .
\end{aligned}
$$

Similarly, for any natural number $\ell$,

$$
\begin{aligned}
d\left(\phi(s, z), \phi\left(t_{n}+(k+\ell) T+s, z\right)\right) \leq & d(\phi(s, z), \phi(s+\ell T, y)) \\
& +d\left(\phi(s+\ell T, y), \phi\left(t_{n}+(k+\ell) T+s, z\right)\right) .
\end{aligned}
$$

Since from the $T$-periodicity of $y$ we get

$$
d(\phi(s, z), \phi(s+\ell T, y))=d(\phi(s, z), \phi(s, y))<\frac{\varepsilon}{2}
$$

and by stability we have

$$
d\left(\phi(s+\ell T, y), \phi\left(t_{n}+k T+\ell T+s, z\right)\right)<\frac{\varepsilon}{2},
$$

and the above conditions hold for every natural number $\ell$, we conclude that

$$
d\left(\phi(s, z), \phi\left(t_{n}+k T+s, z\right)\right)<\varepsilon
$$

holds for every natural $k$. Note that in the beginning we have fixed $k$, while the above says we can take any $k$ without changing the estimation.

We have just shown that

$$
\phi\left(t_{n}+k T, \phi(s, z)\right) \in B(\phi(s, z), \varepsilon) \quad \text { for every } k \in \mathbb{N} \text { and any } s \geq 0,
$$

therefore for any $s \geq 0$ the value $W(\phi(s, z), \varepsilon)$ is bounded by $T$. It follows that $\operatorname{GAP}(z) \leq T$ and thus $\operatorname{GAP}(x) \leq T$. The proof is completed. 
Corollary 4.4

Under the assumptions of Theorem 4.3. if $\omega(x)$ contains a stationary point, then the limit set is the stationary point.

We now present a strengthened version of Theorem 4.1. In particular, it allows to obtain a new proof of Theorem 4.3 Note that in the theorem below the word "positively" (the one related to the notion of asymptotically periodic-like motion) is removed.

\section{THEOREM 4.5}

Let $\phi$ be a dynamical system on a metric space $(X, d)$ and $x \in X$. If $y \in \omega(x)$ is stationary or periodic and positively Lyapunov stable, then $x$ is asymptotically periodic.

Proof. The set $o(y)$ is a compact and minimal set in $\omega(x)$. It follows that $x$ is proximal to some point $p \in o(y)$, that is there exists a sequence $\left(t_{n}\right)_{n \in \mathbb{N}}$ such that

$$
d\left(\phi\left(t_{n}, x\right), \phi\left(t_{n}, p\right)\right) \rightarrow 0 \quad(n \rightarrow+\infty) .
$$

Notice that Lyapunov stability and periodicity of $y$ implies that all points in $o(y)$ are uniformly Lyapunov stable. In particular, for any $\varepsilon>0$ there is $\delta>0$ such that if $d(z, v)<\delta$ for $z \in o(y)$ and $v \in o^{+}(x)$, then $d(\phi(t, z), \phi(t, v))<\varepsilon$ for any $t \geq 0$.

For any $\varepsilon>0$ we find $n(\varepsilon)$ such that $d\left(\phi\left(t_{n(\varepsilon)}, x\right), \phi\left(t_{n(\varepsilon)}, p\right)<\delta\right.$, where $\delta$ corresponds to $\varepsilon$ in the uniform Lyapunov stability condition. This implies that $d(\phi(t, x), \phi(t, p))<\varepsilon$ for any $t>t_{n(\varepsilon)}$. We have shown that $d(\phi(t, x), \phi(t, p)) \rightarrow 0$ as $t \rightarrow+\infty$.

Proof of Theorem 4.3. By Theorem 4.5. $x$ is asymptotically periodic. Then by Theorem 3.8 it is also G-asymptotically periodic.

The stability property described in the above results keeps two points close to each other. In some way one can think of it as some sort of sufficiently good convergence of the orbit of $x$ to the periodic orbit. The example below reveals a different (weaker) kind of convergence to a periodic orbit.

EXAMPLE 4.6

Consider the dynamical system on the plane consisting of a circle and a spiral converging to it from outside. The motion on the spiral is in the clockwise direction with the angular speed greater than 1, decreasing to 1 as the spiral approaches the circle. It is clear that one can assign the speed on the spiral in such a way that the Lyapunov stability in the set $\overline{o^{+}(x)}$ does not hold and the orbit is not asymptotically periodic, but the asymptotic $2 \pi$-periodicity holds (and even Gasymptotic periodicity). The corresponding assignment can be obtained via any decreasing sequence $\left(b_{n}\right)_{n \in \mathbb{N}}$ of positive numbers such that the series generated by the sequence is divergent. Then we take the angular speed to be equal to $1+b_{n}$, where each time the spiral approaches the circle and does one revolution around the center of the circle, $n$ increases by 1 and so $b_{n}$ decreases. 
The rate of convergence in the above example is too slow. This results in lack of the asymptotic periodicity property and leads us to proposing the following criteria.

THEOREM 4.7

Let $\phi$ be a dynamical system on a complete metric space $(X, d)$ and $x \in X$. If

$$
\int_{0}^{+\infty} d(\phi(t+T, x), \phi(t, x)) d t<+\infty
$$

then $x$ is asymptotically T-periodic.

To prove the criteria we use the following characterization of asymptotically $T$-periodic points.

LEMMA 4.8 ([6])

Let $\phi$ be a dynamical system on a complete metric space $(X, d)$. The motion through $x \in X$ is asymptotically $T$-periodic if and only if the sequence $(\phi(n T, x))_{n \in \mathbb{N}}$ converges.

Proof of Theorem 4.7. By the definition of the integral we have

$$
\sum_{n=0}^{+\infty} \max _{t \in[\delta n, \delta(n+1)]} d(\phi(t+T, x), \phi(t, x))<+\infty
$$

for some sufficiently small $\delta>0$. It follows that

$$
\sum_{n=0}^{+\infty} d(\phi(n T+T, x), \phi(n T, x))<+\infty
$$

In particular, the sequence $A:=(\phi(n T, x))_{n \in \mathbb{N}}$ is Cauchy and $x$ is positively asymptotically periodic. The closure of $A$ can either be the same set $A$ or be equal to $A \cup\{p\}$, where $p$ is the only accumulation point of this sequence. The first case implies that $x$ is a periodic orbit while the second case yields

$$
\phi(n T, x) \rightarrow p \quad(n \rightarrow+\infty) .
$$

We can now use Lemma 4.8 to conclude that $x$ is asymptotically $T$-periodic.

Acknowledgments. I thank the Referees for all suggestions that helped improving this paper. I especially thank for all comments regarding Section 4

\section{References}

[1] Bhatia, Nam Parshad and Giorgio P. Szegö. Stability theory of dynamical systems. Vol 161 of Die Grundlehren der mathematischen Wissenschaften. New York, Berlin: Springer-Verlag, 1970. Cited on 45

[2] Birkhoff, George D. Dynamical Systems. Vol. 9 of Amer. Math. Soc. Colloquium Publications. New York: American Mathematical Society, 1927. Cited on 45 
[3] Bohr, Harald. "Zur theorie der fast periodischen funktionen. I. Eine verallgemeinerung der theorie der fourierreihen". Acta Math. 45, no. 1 (1925): 29-127. Cited on 45

[4] Bohr, Harald. "Zur Theorie der Fastperiodischen Funktionen. II. Zusammenhang der fastperiodischen Funktionen mit Funktionen von unendlich vielen Variabeln; gleichmässige Approximation durch trigonometrische Summen." Acta Math. 46, no. 1-2, (1925): 101-214. Cited on 45

[5] Bohr, Harald. "Zur Theorie der fastperiodischen Funktionen. III. Dirichletentwicklung analytischer Funktionen." Acta Math. 47, no. 3 (1926): 237-281. Cited on 45.

[6] Cheban, David N. Asymptotically almost periodic solutions of differential equations. New York: Hindawi Publishing Corporation, 2009. Cited on 45 46$] 53$ and 56 .

[7] Gryszka, Karol. "Asymptotic period in dynamical systems in metric spaces." Colloq. Math. 139, no. 2 (2015): 245-257. Cited on 45 46, 47 and 48

[8] Gryszka, Karol. "Lagrange stability and asymptotic periods." Topology Appl. 204 (2016): 168-174. Cited on 45

[9] Pelczar, Andrzej. "Asymptotically periodic motions." Bull. Polish Acad. Sci. Math. 33, no. 5-6 (1985): 313-319. Cited on 45 46 and 53

[10] Pelczar, Andrzej. "Remarks on some generalizations of asymptotic periodicity in dynamical systems on metric spaces." Ann. Polon. Math. 51 (1990): 259-268. Cited on 45

[11] Pelczar, Andrzej. "Remarks on some recurrency type and periodicity-like conditions in dynamical systems." Univ. Iagel. Acta Math. 35 (1997): 95-113. Cited on 45 .

\author{
Institute of Mathematics \\ Pedagogical University of Cracow \\ Podchorażych str. 2 \\ 30-084 Kraków \\ Poland \\ E-mail: karol.gryszka@up.krakow.pl
}

Received: January 18, 2018; final version: April 24, 2018; available online: May 18, 2018. 